\title{
Comparison of $Q_{\mathrm{C}}^{-1}$ Estimates from Coda Envelopes Constructed from One- and Multi-Component Seismograms
}

\author{
Yutaka Mamada* and Hiroshi Takenaka \\ Department of Earth and Planetary Sciences, Faculty of Science, \\ Kyushu University, Fukuoka 812-8581, Japan
}

S-wave coda is a useful tool to investigate high-frequency seismic wave attenuation in the lithosphere. The generation and amplitude decay of S-wave coda were introduced by a single scattering model proposed by Aki and Chouet (1975). The amplitude decay rate with time is defined by the quality factor of the S-wave coda $\left(Q_{\mathrm{C}}^{-1}\right)$, which is investigated widely at various regions by the single scattering model.

It is only one-component seismograms that have been used to estimate $Q_{\mathrm{C}}^{-1}$ in most studies. This is based on the assumption that the decay of the S-wave coda amplitude recorded on a one-component seismometer is equivalent to the decay of the S-wave coda amplitude which is introduced by the single scattering model. In the single scattering model, S-wave coda is represented by the superposition of single scattered waves which are generated by scatterers distributed randomly and come from any direction to the station. We hereafter call the S-wave coda amplitude expected by the single scattering model the "real coda amplitude." The ground motion of the S-wave coda portion, which consists of the superposition of the scattered waves, has various directions with time. Since the amplitude of the S-wave coda part on a one-component seismogram represents only the projection of the real coda amplitude in the direction of the component, the amplitude on the seismogram depends on the direction of the ground motion. Therefore, it is not trivial that the amplitude decay of a one-component seismogram for a coda part gives the decay of the real coda amplitude (i.e., the assumption). If we have three-component seismograms, we can estimate the real coda amplitude more exactly by vector addition of the amplitudes of the three-component seismograms (hereafter "total vector-amplitude"). In this report, we will check the assumption using real three-component seismic data.

We estimate $Q_{\mathrm{C}}^{-1}$ from a total vector-amplitude and amplitudes of one-component seismograms, and compare them to investigate $Q_{\mathrm{C}}^{-1}$ differences among these amplitudes. Here, we adopt 5 kinds of amplitudes to estimate $Q_{\mathrm{C}}^{-1}$ as a one-component amplitude: three of them are the amplitudes of each original component and the other two are the amplitudes of the radial and transverse components, which are constructed by horizontal two-component amplitudes. Furthermore, we produce the horizontal vector-amplitude, by vector addition of the amplitudes for, horizontal two-component seismograms, and measure $Q_{\mathrm{C}}^{-1}$. This $Q_{\mathrm{C}}^{-1}$ is also compared with the $Q_{\mathrm{C}}^{-1}$ measured from one-component amplitudes or total vector-amplitude.

Received December 16, 1997; Accepted January 30, 1998

* To whom correspondence should be addressed. 
Now, we refer to the data used in this analysis. We use seismograms recorded by three-component velocity type seismometers. Each seismometer records up-down (UD), north-south (NS) and east-west (EW) components of the ground motion. The events analyzed in this report are 78 local ones with magnitudes ranging from 3.0 to 4.6 , which occurred in the period from January 1985 to December 1990 in the Hidaka area of southern Hokkaido. The seismograms were collected at the seismic stations of the Research Center for Earthquake Prediction (RCEP) of Hokkaido University by shortperiod $(2 \mathrm{~Hz})$ velocity seismometers with digital recording at 46 samples per second. In Fig. 1, we show the locations of the four stations and the epicenters of the events used for this study. The epicenters were determined by RCEP.

The amplitudes of the radial and transverse components, and horizontal and total vector-amplitudes are constructed from the three-component seismograms. These amplitudes are given by

$$
A_{\mathrm{R}}=A_{\mathrm{N}} \cos \theta+A_{\mathrm{E}} \sin \theta
$$

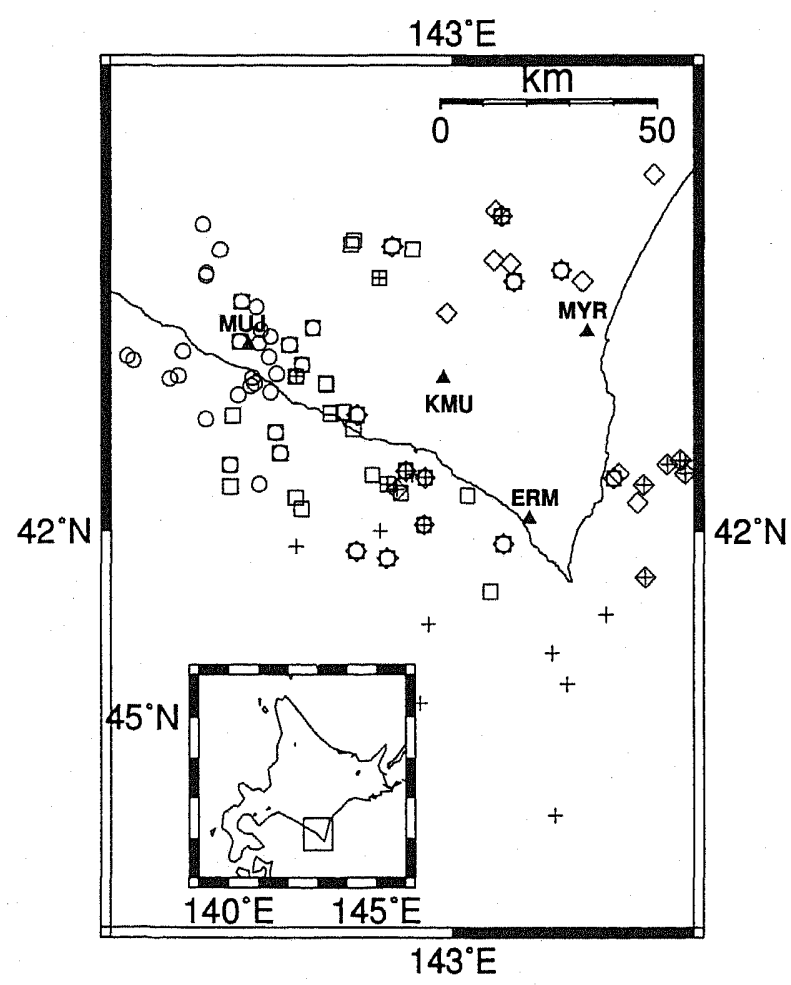

Fig. 1. A map of epicentral distribution of the events used in this report (determined by RCEP). Symbols plotted by square, circle, cross, and diamond represent the epicenters analyzed at KMU, MUJ, ERM, and MYR stations, respectively. These stations are also plotted by the solid triangles with station codes. 
for the radial component,

$$
A_{\mathrm{T}}=-A_{\mathrm{N}} \sin \theta+A_{\mathrm{E}} \cos \theta
$$

for the transverse component,

$$
A_{\mathrm{H}}=\sqrt{A_{\mathrm{R}}^{2}+A_{\mathrm{T}}^{2}}
$$

for the horizontal vector-amplitude and

$$
A_{t}=\sqrt{A_{\mathrm{Z}}^{2}+A_{\mathrm{R}}^{2}+A_{\mathrm{T}}^{2}}
$$

for the total vector-amplitude; where $A_{\mathrm{Z}}, A_{\mathrm{N}}$, and $A_{\mathrm{E}}$ represent the amplitudes of the $\mathrm{UD}, \mathrm{NS}$, and EW components, respectively. Here, $\theta$ is an azimuthal angle defined by the angle between vectors pointed to the north and to the station measured clockwise from the epicenter. The azimuthal angle $(\theta)$ is necessary to estimate the amplitudes of the radial and transverse components in Eqs. (1) and (2). For estimating the azimuthal angle, we use the epicentral location of each event determined by RCEP. On the other hand, for the horizontal and total vector-amplitudes, since the relation

$$
A_{\mathrm{R}}^{2}+A_{\mathrm{T}}^{2}=A_{\mathrm{E}}^{2}+A_{\mathrm{N}}^{2}
$$

holds, we can estimate these amplitudes without estimation of the azimuthal angles. We calculate $A_{\mathrm{H}}$ and $A_{t}$ by use of Eq. (5).

We use the single isotropic scattering model extended by Sato (1977) for the configuration that a station and hypocenter are at different places. Based on this model, the logarithm of the coda amplitude is represented in terms of the frequency and lapse time measured from the event origin time as

$$
\begin{aligned}
& \ln \left[A(\omega, t) r / \sqrt{K\left(V_{\mathrm{S}} t / r\right)}\right]=\ln (C(\omega))-\left(\omega / 2 Q_{\mathrm{C}}\right) t \\
& K(x)=(1 / x) \ln [(x+1) /(x-1)] \quad \text { for } \quad x>1 ;
\end{aligned}
$$

where $A(\omega, t), \omega, t, r, V_{\mathrm{S}}$ and $C(\omega)$ are bandpass-filtered coda amplitude, angular frequency, lapse time, source-receiver distance, $S$-wave velocity, and the term related to source, respectively. $A(\omega, t)$ for the UD, NS, and EW components are estimated by filtering the original seismograms for a narrow frequency band centered at 1.0, 2.0, 4.0, 8.0 , and $16.0 \mathrm{~Hz}$ with a bandwidth of an octave, and calculating RMS (the root mean square) on a temporary window of 4 cycles which shifts at steps of 1 cycle throughout the S-wave coda at each frequency band. For the radial and transverse components, after constructing a seismogram for each component by Eqs. (1) or (2), $A(\omega, t)$ is calculated by RMS mentioned above. For the $A(\omega, t)$ of horizontal and total vectoramplitudes, we apply a bandpass filter to the original three-component seismograms, estimate each amplitude by Eqs. (3), (4), and (5), and calculate $A(\omega, t)$ by RMS. After all, we get 7 values of $Q_{\mathrm{C}}^{-1}$ for each event at each station, which are estimated from the amplitudes of the UD, NS, EW, radial and transverse components, and horizontal and total vector-amplitudes.

We consider two cases for the time-window selection to estimate $Q_{\mathrm{C}}^{-1}$. In the first case, we choose the time window from twice the $S$-wave arrival time to the time when 
amplitude decays to three times the noise level for each frequency range. This timewindow regime is widely used in the study of $Q_{\mathrm{C}}^{-1}$ analysis (Aki and Chouet, 1975; Tsujiura, 1978; Sato et al., 1988). In this study, the noise level is determined from the amplitude for $5 \mathrm{~s}$ before the $\mathrm{P}$-wave arrival. In the second case, the time window is fixed at a lapse time measured from event origin time for all events, taking into account $Q_{\mathrm{C}}^{-1}$ dependence on the lapse time. The details for this time-window selection for each station will be mentioned in the following.

Figure 2 shows the envelopes of S-wave coda given by the term of the left-hand side of Eq. (6), which have been constructed from 5 kinds of one-component amplitudes, and horizontal and total vector-amplitudes, respectively. We can see that the envelope fluctuations of the horizontal and total vector-amplitudes are smaller than those of individual one-component amplitude, and that the envelopes for the total vector-

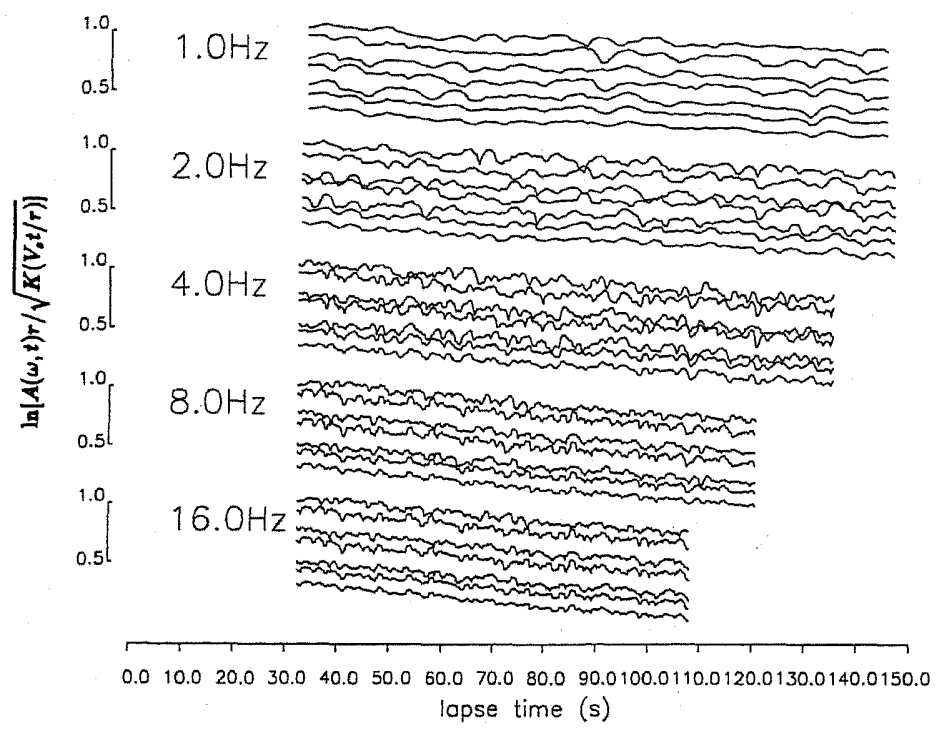

Fig. 2. Example of envelopes of S-wave coda given by the term of the left-hand side of Eq. (6) for an event observed at KMU. The envelopes are represented for five frequency bands $(1.0,2.0,4.0,8.0$, and $16.0 \mathrm{~Hz})$. There are seven traces for each frequency band, which are the envelopes of the UD, NS, EW, radial, and transverse components, and horizontal and total vector-amplitudes from top to bottom. Each trace is normalized by the corresponding maximum value of the envelope of the total vector-amplitude for each frequency band, and shifted along the vertical axis. The lapse time measured from the event origin time is shown at the bottom of the figure. One should notice that the envelope fluctuations of the horizontal and total vector-amplitudes are smaller than those of the amplitude for any one-component seismogram. Especially, the fluctuation of the total vector-amplitude is the smallest among all the kinds of amplitudes at all frequency rariges. 
amplitude decay smoothly as expected by the single scattering model. Since $Q_{\mathrm{C}}^{-1}$ can be evaluated by fitting the right-hand side of Eq. (6) to the envelopes shown in Fig. 2, we expect that the coda envelopes measured from the total vector-amplitudes give more stable $Q_{\mathrm{C}}^{-1}$ (i.e., the standard deviation of $Q_{\mathrm{C}}^{-1}$ is smaller) than those measured from any other amplitude.

Figure 3 shows $Q_{\mathrm{C}}^{-1}$ estimated from all events for each kind of amplitude at KMU where the number of analyzed events is the largest among all stations. Here the time window has been taken by the first case of time-window selection. The end of the time window for the horizontal or total vector-amplitude has been decided by the time when the RMS amplitude for individual (horizontal or total) bandpass-filtered amplitude of coda part decays to three times the RMS amplitude for individual bandpass-filtered amplitude before P-wave arrival. For the same event, the time windows for all kinds of amplitudes were almost the same after all. Figure 3 indicates that $Q_{\mathrm{C}}^{-1}$ estimated from the total vector-amplitudes tends to be less scattered than that from only onecomponent amplitudes. The maximum value of $Q_{\mathrm{C}}^{-1}$ is, at most, about twice the minimum value of $Q_{\mathrm{c}}^{-1}$ for the total vector-amplitude. On the contrary, the maximum value is always greater than twice the minimum value for some one-component amplitudes.

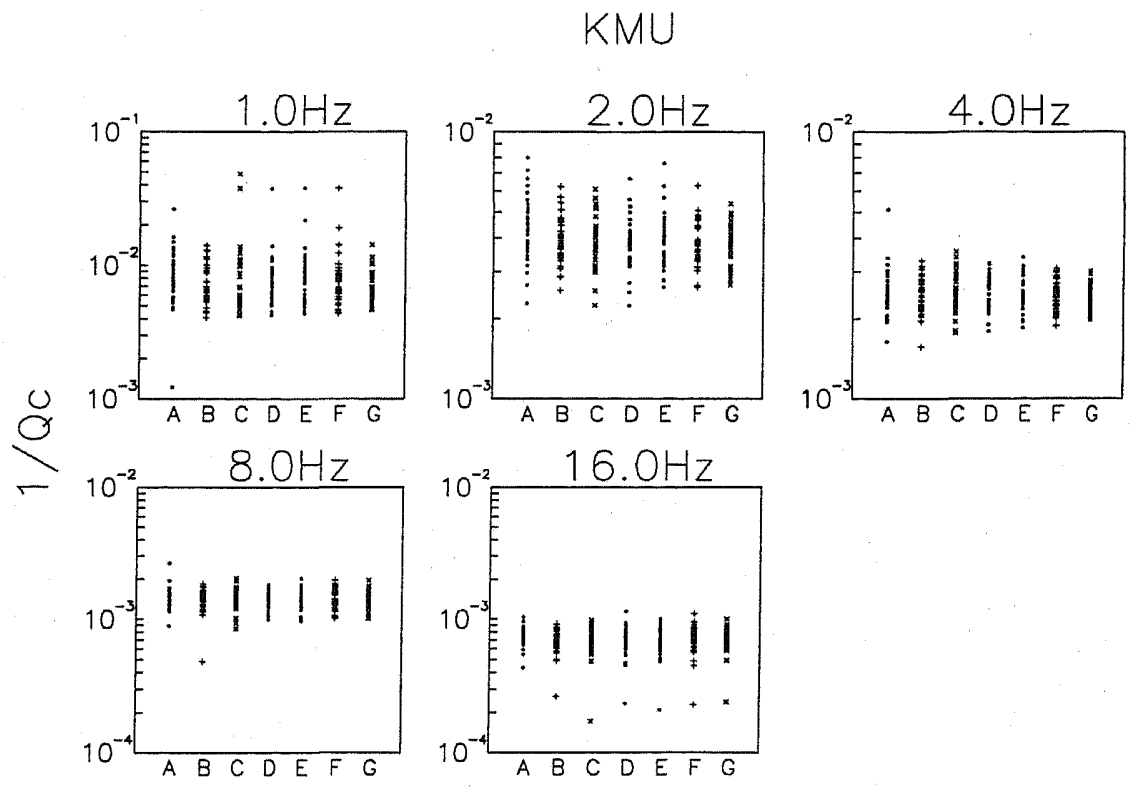

Fig. 3. Distribution of the estimated $Q_{\mathrm{C}}^{-1}$ for the five frequency ranges at $\mathrm{KMU}$ station. The labels from $A$ to $G$ along the horizontal axis correspond to the amplitudes of the UD, NS, EW, radial, and transverse components, and the horizontal and total vector-amplitudes, respectively. Each symbol represents the $Q_{\mathrm{C}}^{-1}$ value estimated from each event. The time window has been defined by first time-window selection (see text). One should notice that the estimated $Q_{\mathrm{C}}^{-1}$ for the total vector-amplitude tends to be less scattered than that for any other amplitude, which was also observed at the other stations.

Vol. 45, No. 6, 1997 
Table 1. Ratios of the standard deviations of $Q_{\mathrm{C}}^{-1}$ to the estimated $Q_{\mathrm{C}}^{-1}$ averaged over all events and stations. The unit is percent. "Horizontal" and "Total" in the first column mean the horizontal and total vector-amplitudes, respectively.

\begin{tabular}{lccccc}
\hline & $1.0 \mathrm{~Hz}$ & $2.0 \mathrm{~Hz}$ & $4.0 \mathrm{~Hz}$ & $8.0 \mathrm{~Hz}$ & $16.0 \mathrm{~Hz}$ \\
\hline UD & 13.1 & 8.8 & 5.2 & 3.5 & 2.3 \\
NS & 14.2 & 8.1 & 5.2 & 3.2 & 2.2 \\
EW & 14.3 & 7.5 & 5.4 & 3.0 & 2.0 \\
Radial & 15.5 & 8.1 & 5.1 & 3.2 & 2.1 \\
Transverse & 14.4 & 8.3 & 5.8 & 3.3 & 2.2 \\
Horizontal & 10.2 & 7.1 & 4.1 & 2.2 & 1.4 \\
Total & 9.3 & 6.2 & 3.2 & 2.1 & 1.2 \\
\hline
\end{tabular}

Comparing $Q_{\mathrm{C}}^{-1}$ estimated from the 5 kinds of one-component amplitudes, there are differences in the distribution among them. Such differences in the distribution of the estimated $Q_{\mathrm{C}}^{-1}$ for different kinds of amplitudes were also found at the other stations.

To check the stability of the estimated $Q_{\mathrm{C}}^{-1}$ from every event for each kind of amplitude, we calculate the fraction of the standard deviation in estimated $Q_{\mathrm{C}}^{-1}$ for every event, and compare the fractions among all kinds of amplitude. The fraction is estimated as follows. First, we measure the ratio of the standard deviation of $Q_{\mathrm{C}}^{-1}$ to the estimated value of $Q_{\mathrm{C}}^{-1}$ for each event in each kind of amplitude. Next, we average. the ratios over all events for each kind of amplitude. These averaged ratios may represent the goodness of the model fitting for each kind of amplitude. The results are shown in Table 1. It shows that the ratios for the vector-amplitude are much smaller than those for the 5 kinds of one-component amplitudes for all frequency ranges. In some cases, the ratio for the total vector-amplitude is approximately only half the ratios for the one-component amplitudes. This result shows that, for every event, $Q_{\mathrm{C}}^{-1}$ given by the total vector-amplitude is more stable than that by any other amplitude. It also means the coda envelopes obtained from the one-component amplitudes suffer from much more fluctuation than that from the total vector-amplitude. The ratios of the standard deviations of $Q_{\mathrm{C}}^{-1}$ to the values of $Q_{\mathrm{C}}^{-1}$ estimated from the horizontal vectoramplitudes are also shown in Table 1 . They are larger than those for the total vectoramplitude, but smaller than those for the one-component amplitudes. In other words, the envelope fluctuation can be reduced much more by the use of the horizontal vectoramplitude than by the use of any one-component amplitude. However, the fluctuation is still larger than that for the total vector-amplitude. Thus, we recognize that the envelope fluctuation for the total vector-amplitude is the smallest among that for all of the amplitudes used in this study.

When we evaluate $Q_{\mathrm{C}}^{-1}$ in any region, it is usual to average $Q_{\mathrm{C}}^{-1}$ over many events. We estimate the mean value of $Q_{\mathrm{c}}^{-1}, \mu$, by the following equation: 


$$
\mu=\frac{\sum_{i=1}^{N} w_{i} x_{i}}{\sum_{i=1}^{N} w_{i}},
$$

and the standard deviation of $Q_{\mathrm{C}}^{-1}, \sigma$, by

$$
\sigma=\sqrt{\frac{\sum_{i=1}^{N} w_{i}\left(x_{i}-\mu\right)^{2}}{\sum_{i=1}^{N} w_{i}} \times \frac{N}{N-1}} ;
$$

where $N$ is the number of samples to be averaged. $x_{i}$ and $w_{i}$ represent

$$
x_{i}=\left(Q_{\mathrm{C}}^{-1}\right)_{i}
$$

and

$$
w_{i}=\frac{1}{\sigma_{i}^{2}} ;
$$

where $\left(Q_{\mathrm{C}}^{-1}\right)_{i}$ and $\sigma_{i}$ are the value of $Q_{\mathrm{C}}^{-1}$ and standard deviation of $Q_{\mathrm{C}}^{-1}$ estimated from the seismic records of the $i$-th event, respectively. The result is shown in Fig. 4. From this figure, we find the differences of the mean $Q_{\mathrm{C}}^{-1}$ among all kinds of amplitudes are small. The difference between the largest and smallest mean values of $Q_{\mathrm{C}}^{-1}$ among the 7 kinds of amplitudes is much less than a tenth of the estimated mean value of $Q_{C}^{-1}$ in each frequency band. For example at KMU, the orders of the differences are $10^{-4}$ at 1.0 and $2.0 \mathrm{~Hz}$ bands, and $10^{-5}$ at the other frequency bands. These are less than $10 \%$ of the estimated mean values of $Q_{\mathrm{C}}^{-1}$ for any kind of amplitude. On the other hand, the standard deviations of $Q_{\mathrm{C}}^{-1}$ range between 10 and $30 \%$ of the mean values of $Q_{\mathrm{C}}^{-1}$. For the other stations, the results are almost the same as that for KMU. Therefore, in the first case of time-window selection, we can conclude that the values of $Q_{\mathrm{C}}^{-1}$ estimated from different kinds of amplitudes are almost equal within the standard deviations.

Comparing the magnitudes of the standard deviations of $Q_{\mathrm{C}}^{-1}$ estimated from the 5 kinds of one-component amplitudes, they are different depending on which kind of amplitude we select to estimate them. Moreover, if we select the component for which the standard deviation is the smallest among the 5 kinds of one-component amplitudes, the selected component differs with frequency. For example at MYR, the component giving the smallest standard deviation is the radial component (labeled with $\mathrm{D}$ in Fig. 4) for $1.0 \mathrm{~Hz}$ band, EW component (labeled with C) for 2.0 and $16.0 \mathrm{~Hz}$ bands, and UD component (labeled with A) for 4.0 and $8.0 \mathrm{~Hz}$ bands. This means that if we use a one-component amplitude to estimate $Q_{\mathrm{C}}^{-1}$, the accuracy of the estimated $Q_{\mathrm{C}}^{-1}$ value depends on which kind of amplitude we select among the 5 kinds of one-component amplitudes. On the contrary, the standard deviation for the total vector-amplitude is almost the same or less than the smallest one among the 5 kinds of one-component 

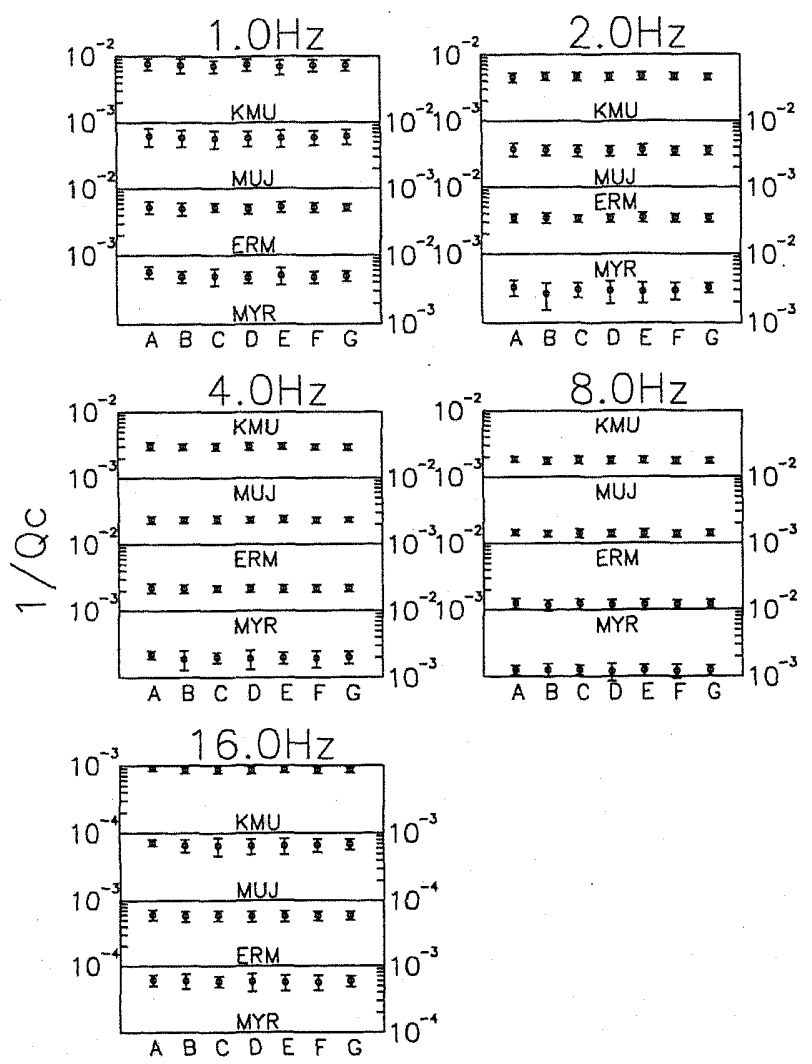

Fig. 4. Mean $Q_{\mathrm{C}}^{-1}$ and the standard deviations estimated by Eqs. (7) and (8) at the four stations. The labels from $A$ to $G$ along the horizontal axis are described in the caption to Fig. 3. The time window has been defined by first time-window selection. Note that although the estimated $Q_{\mathrm{C}}^{-1}$ among all the kinds of amplitudes are almost the same within the standard deviations, the standard deviation for the total vector-amplitude is smaller than that for any other amplitude.

amplitudes for all frequency bands. As a result, $Q_{\mathrm{C}}^{-1}$ measurement using the total vector-amplitude is the most reliable for all frequency bands.

Next, we attend to $Q_{\mathrm{C}}^{-1}$ measured by the second case of time-window selection. In the first case, the time window for $Q_{C}^{-1}$ measurements was different for every event. There are some studies which show the selection of time window can affect the estimated $Q_{\mathrm{C}}^{-1}$ value. Some researchers found that $Q_{\mathrm{C}}^{-1}$ estimated from an early part of the coda are greater than the $Q_{\mathrm{C}}^{-1}$ from the later part (Rautian and Khalturin, 1978; Roecker et al., 1982; Pulli, 1984; Kosuga, 1992). The same phenomenon can be also predicted numerically, since the later part of the coda is more affected by multiple scattering than the early part (Gao et al., 1983; Hoshiba, 1991). In order to restrict the effects of time-window selection on the estimation of $Q_{\mathrm{C}}^{-1}$ as much as possible, we now measure 
$Q_{\mathrm{C}}^{-1}$ with a common time window for all events (i.e., the estimation of $Q_{\mathrm{C}}^{-1}$ by the second time-window selection).

A time window common to all records at each station is selected so that the largest number of events in Fig. 1 could be analyzed under the condition that the coda portion used to estimate $Q_{\mathrm{C}}^{-1}$ could be taken for at least $20 \mathrm{~s}$. As a result, the selected time windows for $Q_{\mathrm{C}}^{-1}$ measurements were from 25 to $45 \mathrm{~s}$ measured from the event origin time at KMU and from 20 to $40 \mathrm{~s}$ at MUJ. Since a small number of events remained if we selected the common time window for all events at ERM and MYR, we did not evaluate $Q_{\mathrm{C}}^{-1}$ at these stations. The result at KMU is plotted in Fig. 5. The scatter of measured values of $Q_{\mathrm{C}}^{-1}$ for the events is less for the total or horizontal vector-amplitude than for the one-component amplitudes as well as for the first case of time-window selection. The averaged $Q_{\mathrm{C}}^{-1}$ over all events and standard deviations of $Q_{\mathrm{C}}^{-1}$ at each station are shown in Fig. 6. This figure shows that $Q_{\mathrm{C}}^{-1}$ for the one-component amplitudes and total vector-amplitude are almost the same within the standard deviations again. However, the standard deviation for the total vector-amplitude is the smallest among any kind of amplitude in most frequency bands, and it is from half to two-thirds that of the largest one among the 5 kinds of one-component amplitudes. It was found that total vector-amplitude can give more stable $Q_{\mathrm{C}}^{-1}$ than any one-component amplitude for the second case of time-window selection as well as the first case.

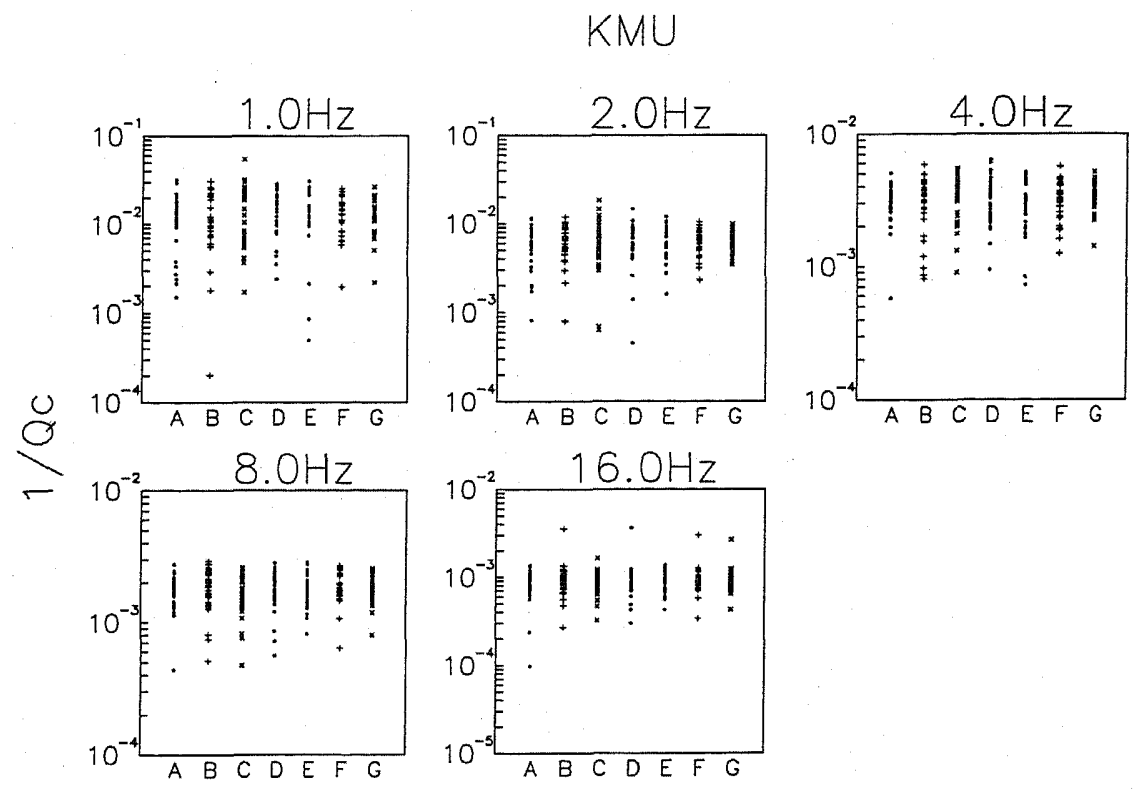

Fig. 5. Distribution of the estimated $Q_{\mathrm{C}}^{-1}$ for the five frequency ranges at KMU station. The labels from $A$ to $G$ along the horizontal axis are described in the caption to Fig. 3. The time window used for the $Q_{\mathrm{c}}^{-1}$ estimation is from 25 to $45 \mathrm{~s}$ measured from the event origin time for all events. One should notice that the estimated $Q_{\mathrm{c}}^{-1}$ for the total vector-amplitude tends to be less scattered than that for any other amplitude, as can be seen in Fig. 3.

Vol. 45 , No. 6,1997 

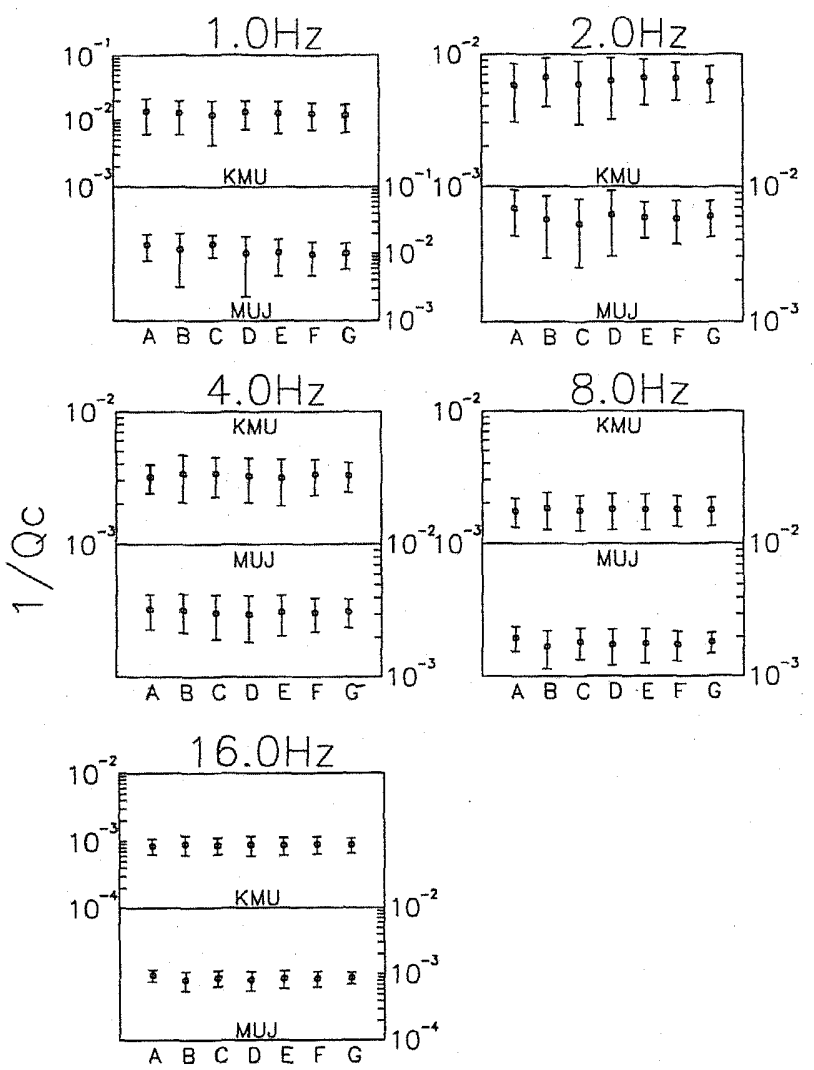

Fig. 6. Mean $Q_{\mathrm{C}}^{-1}$ and standard deviations estimated by Eqs. (7) and (8) at stations KMU and MUJ. The labels from $A$ to $G$ along the horizontal axis are described in the caption to Fig. 3. The time windows used for the $Q_{\mathrm{C}}^{-1}$ estimation are from 25 to $45 \mathrm{~s}$ for KMU and 20 to $40 \mathrm{~s}$ for MUJ measured from the event origin time. Note that although the estimated $Q_{\mathrm{C}}^{-1}$ among all the kinds of amplitudes are almost the same within the standard deviations, the standard deviation for the total vector-amplitude is the smallest among them in most frequency bands.

Comparing the distribution of $Q_{\mathrm{C}}^{-1}$ for each kind of amplitude in the second case of time-window selection with that in the first case (Figs. 3 and 5), the former is about twice larger than the latter. This fact is seen even for the total and horizontal vectoramplitudes. We should notice that time-window selection is important to evaluate stable $Q_{C}^{-1}$ even if we use the coda envelope for the total vector-amplitude.

Now we discuss the result that the envelope fluctuation of the total vector-amplitude is smaller than that of individual one-component amplitude as mentioned before. Kennett (1993) has employed the total vector-amplitude to investigate the dependence of the amplitude of not S-wave coda but the $\mathrm{Pn}, \mathrm{Sn}$, and $\mathrm{Lg}$ phases as a function of range from the source to $700 \mathrm{~km}$ with a linear array for an explosion source in water. 
He showed the amplitude behaviors for these phases, as a function of range, can be discerned in cartesian components but are clearer in vector representation, because the amplitude distribution versus epicentral distance in vector representation is less scattered. He pointed out, for this reason, that the amplitude given by the vector measurement does not depend on the incident direction of these phases, since its amplitude is invariant for the rotation of the coordinate. This means that even if we cannot have the knowledge of the azimuthal or dip angle of the incident wave (i.e., the knowledge of the direction of the motion of the incident wave), the amplitude of the incident wave can be measured correctly by the total vector-amplitude of threecomponent seismograms in the rectangular coordinate system. Turning now to S-wave coda, the amplitude can always be measured by total vector-amplitude more correctly than by one-component amplitude, since the motion of S-wave coda may have any direction with time. On the other hand, the horizontal vector- or one-component amplitude in a direction represents only the projection of the coda amplitude on the horizontal plane or direction. Hence, these kinds of amplitudes depend on not only the coda amplitude but also the direction of the motion of the coda waves. The apparent fluctuation resulting from the variation of the direction of the S-wave coda motion must be included in the envelopes of these kinds of amplitudes.

We now summarize the results made clear in this study.

(1) The coda envelopes obtained from the one-component amplitudes suffer from much more fluctuation than that from total-vector amplitude.

(2) There are more variations in $Q_{\mathrm{C}}^{-1}$ estimated from one-component amplitudes than those estimated from total vector-amplitudes.

(3) The mean values of $Q_{\mathrm{C}}^{-1}$, which are obtained by averaging overall events at each station, for the horizontal and total vector-amplitudes and one-component amplitudes are almost the same within the standard deviations. However, the standard deviation for the total vector-amplitude is the smallest among all the kinds of amplitudes in most frequency bands.

The above results suggest that the result of $Q_{\mathrm{C}}^{-1}$ estimated from a one-component seismogram is reliable, however we should use total vector-amplitude to measure more stable and reliable $Q_{C}^{-1}$ if three-component records are available.

We thank Dr. Y. Motoya and Dr. M. Kasahara of the Research Center for Earthquake Prediction of Hokkaido University for providing the waveform data and information for the epicenters. The comments of anonymous referees are helpful for improving the manuscript.

\section{REFERENCES}

Aki, K. and B. Chouet, Origin of coda waves: source, attenuation and scattering effects, $J$. Geophys. Res., 80, 3322-3342, 1975.

Gao, L. S., L. C. Lee, N. N. Biswas, and K. Aki, Comparison of the effects between single and multiple scattering on coda waves for local earthquakes, Bull. Seismol. Soc. Am., 73, 377-389, 1983.

Hoshiba, M., Simulation of multiple scattered coda wave excitation based on the energy conservation law, Phys. Earth Planet. Inter., 67, 123-136, 1991.

Vol. 45, No. 6, 1997 
Kennett, B. L. N., The distance dependence of regional phase discriminants, Bull. Seismol. Soc. Am., 83, 1155-1166, 1993.

Kosuga, M., Dependence of coda $\mathrm{Q}$ on frequency and lapse time in the western Nagano region, Central Japan, J. Phys. Earth, 40, 421-445, 1992.

Pulli, J. J., Attenuation of coda waves in New England, Bull. Seismol. Soc. Am., 74, 1149-1166, 1984.

Rautian, T. G. and V. I. Khalturin, The use of the coda for determination of the earthquake source spectrum, Bull. Seismol. Soc. Am., 68, 923-948, 1978.

Roecker, S. W., B. Tucker, J. King, and D. Hatzfeld, Estimates of Q in central Asia as a function of frequency and depth using the coda of locally recorded earthquakes, Bull. Seismol. Soc. Am., 72, 129-149, 1982.

Sato, H., Energy propagation including scattering effects: single isotropic scattering approximation, J. Phys. Earth, 25, 27-41, 1977.

Sato, H., A. M. Shomahmadov, V. I. Khalturin, and T. G. Rautian, Temporal change in spectral coda attenuation $Q_{\mathrm{C}}^{-1}$ associated with the $K=13.3$ earthquake of 1983 near Garm, Tadjikistan region in Soviet Central Asia, Zisin, 41, 39-46, 1988 (in Japanese with an English abstract).

Tsujiura, M., Spectral analysis of the coda waves from local earthquakes, Bull. Earthq. Res. Inst., Univ. Tokyo, 53, 1-48, 1978. 